\title{
ON INEQUALITIES OF PERIODIC FUNCTIONS AND THEIR DERIVATIVES
}

\author{
Z. DITZIAN
}

\begin{abstract}
The inequality $\|f\|_{B} \leqslant a_{h}\left\|f^{(h)}\right\|_{B}$ is proved for many spaces of periodic
\end{abstract} functions. An analogue for sequences is also given.

1. In his paper [6] in 1939 Northcott proved for periodic functions for which $\int_{0}^{2 \pi} f(x) d x=0$ and $f, \ldots, f^{(k-1)}$ are absolutely continuous that

$$
\begin{aligned}
& \|f\|_{\infty} \leqslant a_{k}\left\|f^{(k)}(\cdot)\right\|_{\infty} \text { where } a_{k}=\frac{4}{\pi} \sum_{i=1}^{\infty} i^{-k-1} \text { for } k \text { odd } \\
& \text { and } a_{k}=\frac{4}{\pi} \sum_{i+1}^{\infty}(-1)^{i+1} i^{-k-1} \text { for } k \text { even }
\end{aligned}
$$

and for each $k, a_{k}$ is best possible and is actually achieved. Bellman [2] later showed that

$$
\|f(\cdot)\|_{2 r} \leqslant a_{k}\left\|f^{(k)}(\cdot)\right\|_{2 r}
$$

holds. Here the constants $a_{k}$ are the same as in (1.1), $r>1$ is an integer, and $f$ is any $2 \pi$-periodic function such that $\int_{0}^{2 \pi} f(x) d x=0, f^{(k-1)}$ is absolutely continuous, and $f^{(k)} \in L_{2 r}$. The constants $a_{k}$ in (1.2) are not best possible in general. For $r=1$ the best constant is 1 by Wirtinger's inequality [1]. We will prove that if $L_{2 r}(T)$ $(T \equiv[0,2 \pi]$ for $f(x)$ satisfying $f(0)=f(2 \pi))$ is replaced by $L_{p}(T), p \geqslant 1$, we still have

$$
\|f(\cdot)\|_{p} \leqslant a_{k}\left\|f^{(k)}(\cdot)\right\|_{p}
$$

and for each $k, a_{k}$ is best possible for $L_{1}$. We shall show that there is a whole class of Banach spaces for which an analogous result to (1.3) is valid.

In $1954 \mathrm{~K}$. Fan, O. Taussky and J. Todd [4] proved a discrete analogue of the above that for a real finite sequence $x_{1}, \ldots, x_{n}, n \geqslant 2$, satisfying $\sum_{i=1}^{n} x_{i}=0$

$$
\begin{aligned}
& \max \left|x_{i}\right| \leqslant \frac{n}{4} \max \left|x_{i+1}-x_{i}\right| \text { for even } n \text { and } \\
& \max \left|x_{i}\right| \leqslant \frac{n^{2}-1}{4} \max \left|x_{i+1}-x_{i}\right| \text { for odd } n
\end{aligned}
$$

Received by the editors April 5. 1982.

1980 Mathematics Subject Classification. Primary 26D10, 26 DI5. 
and the (1.4) estimate is achieved and therefore is best possible. They proved also that for any positive integer $r$ and defining $x_{j} \equiv x_{i}$ if $j=i(\bmod n)$,

$$
\begin{aligned}
& \left\{\sum_{i=1}^{n}\left|x_{i}\right|^{r}\right\}^{1 / r} \geqslant \frac{n-1}{2}\left(\sum_{i=-1}^{n}\left|x_{i+1}-x_{i}\right|^{r}\right)^{1 / r} \text { and } \\
& \left\{\sum_{i=1}^{n}\left|x_{i}\right|^{r}\right\}^{1 / r} \leqslant \frac{n^{2}-1}{12}\left(\sum_{i=1}^{n}\left|x_{i-1}-2 x_{i}+x_{i+1}\right|^{r}\right)^{1 / r}
\end{aligned}
$$

(where $x$, may be complex). We shall prove for $1 \leqslant p<\infty$

$$
\left\{\sum_{i-1}^{n}\left|x_{i}\right|^{p}\right\}^{1 / p} \leqslant b_{n}^{k}\left\{\sum_{i=1}^{n}\left|\Delta^{k} x_{i}\right|^{p}\right\}^{1 / p}
$$

where $b_{n}$ is $n / 4$ or $\left(n^{2}-1\right) / 4$ for even or odd $n$ respectively.

The constants are better than those in (1.5), $p$ is not required to be an integer, and $k$ can be any integer, not just 1 or 2 .

2. The inequality on periodic functions. Using Northcott's result and a technique established in proving inequalities of Kolmogorov type, see [3], we have

THEOREM 2.1. Suppose $f$ is a $2 \pi$ periodic function satisfying $\int_{0}^{2 \pi} f(x) d x=0$ and for which $f, \ldots, f^{(h-1)}$ are absolutely continuous and $f^{(k)} \in L_{p}(T)$ for some $p, 1 \leqslant p<\infty$. Then

$$
\|f\|_{p} \leqslant a_{k}\left\|f^{(k)}\right\|_{p} \text { where } a_{k} \text { is given by (1.1). }
$$

Proof. Let $F(x)=\int_{0}^{2 \pi} f(x+t) g(t) d t$ where $g \in L_{q}, q^{-1}+p^{-1}=1$, and $\|g\|_{q}=$ 1. Obviously $F(x)$ satisfies $\int_{0}^{2 \pi} F(x) d x=0$ and $F^{(i)}(x)$ is absolutely continuous for $i<k$ and $F^{(k)}(x)$ is continuous. Therefore, $|F(0)| \leqslant\|F(x)\|_{\infty} \leqslant a_{k}\left\|F^{(k)}(\cdot)\right\|_{\infty} \leqslant$ $a_{h}\left\|f^{(k)}\right\|_{p}$. Choosing $g$ so that $|F(0)|=\left|\int_{0}^{2 \pi} f(t) g(t) d t\right|=\|f\|_{p}$, we complete the proof.

In fact the following more general theorem can be proved equally easily. The theorem is applicable to Banach space $B$ on which a group of isometries $S(t)$ is defined satisfying $S(2 \pi)=S(0)=I$. We will say that $f \in B$ is in $D(A)$ if the strong limit of $(S(\eta)-I) f / \eta$ as $\eta \rightarrow 0+$ exists, and $f \in D\left(A^{k}\right)$ if $f \in D\left(A^{k-1}\right)$ and $s-\lim _{\eta \rightarrow 0+}(S(\eta)-I) A^{k-1} f / \eta$ exists. We say $f \in D_{n}\left(A^{k}\right)$ if the limit exists in the weak sense and $f \in D_{n^{*}}\left(A^{k}\right)$ if the limit exists in the weak* sense (which is only possible if $B=X^{*}$ ).

THEOREM 2.2. Let $B$ be a Banach space, $S(t)$ a group of isometries and $f \in D\left(A^{k}\right)$ or $f \in D_{n}\left(A^{k}\right)$ or $f \in D_{n \cdot(}\left(A^{k}\right)$, and suppose $\int_{0}^{2 \pi} T(t) f d t=0$ in the weak or weak* sense. Then

$$
\|f\|_{B} \leqslant a_{k}\left\|A^{k} f\right\|_{B} \text { where } a_{k} \text { is given by (1.1). }
$$

Proof. For $f \in D\left(A^{k}\right)$ or $f \in D_{w}\left(A^{k}\right)$ choose $g \in B^{*}$ such that $\|g\|_{B^{*}}=1$ and write $F(x)=\langle S(x) f, g\rangle$. Obviously $F(x), \ldots, F^{(k-1)}(x)$ are absolutely continuous, $F(x)$ periodic and $\int_{0}^{2 \pi} F(x) d x=0$, and therefore

$$
|\langle f, g\rangle|=|F(0)| \leqslant\|F\|_{\infty} \leqslant a_{k}\left\|F^{(k)}(\cdot)\right\|_{\infty} \leqslant a_{k}\left\|A^{k} f\right\| .
$$


Choosing $g$ so that $|\langle f, g\rangle| \geqslant\|f\|_{B}-\varepsilon$, we complete the proof of this case. For $f \in D_{w^{*}}\left(A^{k}\right)$ we choose $g \in X, X^{*}=B$, and proceed similarly.

RemarK. There are many spaces satisfying the above, $L_{p}(T)$ Sobolev spaces, $L_{p}^{r}(T)$, Orlicz spaces on $T$ and others. The weak* limit would be of interest for the space of functions of bounded variation or duals of Sobolev spaces.

THEOREM 2.3. For $f \in L_{1}$ or $\alpha \in B . V$. where $\int_{0}^{2 \pi} f(x) d x=0$ or $\int_{0}^{2 \pi} d \alpha(x)=0$ respectively we have $\|f\|_{1} \leqslant a_{k}\left\|f^{(k)}\right\|_{1}$ or $\|\alpha\|_{B . V .} \leqslant a_{k}\left\|\alpha^{(k)}\right\|_{B . V}$. or $\|f\|_{1} \leqslant$ $a_{k}\left\|f^{(k-1)}\right\|_{B . V}$. where $a_{k}$ is as defined in (1.1) and this $a_{k}$ is best possible here.

Proof. The inequality was proved in Theorem 2.2. What is left is to show that $a_{k}$ is best possible.

The extremum is achieved on a variant of the function given by Northcott. We will use notation and known results from a well-known paper of Kolmogorov [5]. Let

$$
f_{k}(x)=\frac{4}{\pi} \sum_{m=0}^{\infty} \frac{\sin ((2 m+1) x-(\pi / 2) k)}{(2 m+1)^{k+1}} .
$$

Obviously $f_{0}(x)=f_{k}^{(k)}(x),\left\|f_{0}\right\|_{\text {B.V. }[T]}=4$ and

$$
\left\|f_{k}\right\|_{\text {B.V. }[T]}=4 \frac{4}{\pi} \sum_{m=0}^{\infty} \frac{1}{(2 m+1)^{k+1}}
$$

for $k$ odd and

$$
\left\|f_{k}\right\|_{\text {B.V. }[T]}=4 \frac{4}{\pi} \sum_{m=0}^{\infty} \frac{(-1)^{m}}{(2 m+1)^{k+1}}
$$

for $k$ even (see description of $f_{n}[5, \mathrm{p} .236]$ ). For $\alpha(x)=f_{k}(x)$ the equality is achieved. We can, therefore, approximate $f \in L_{1}$ by $f(x)=(1 / h) \int_{x}^{x+h} d \alpha(t)(\alpha(t)$ $=f_{k}(t)$ here) and for small enough $h$ the inequality is almost achieved. For $\alpha(t) \in$ B.V. $(T),\|\alpha\|_{\text {B.V. }} \leqslant a_{k}\left\|\alpha^{(k)}\right\|_{\text {B.V. }}$ and $\|f\|_{L_{1}} \leqslant a_{k}\left\|f^{(k-1)}\right\|_{\text {B.V. }}$ and $a_{k}$ is best possible and the critical element is in the space. For $\|f\|_{L_{1}(T)} \leqslant a_{k}\left\|f^{(k)}\right\|_{l, 1}(T), a_{k}$ is best possible and the critical element is not in the space.

3. The discrete case. In [4] the following theorem was proved.

THEOREM A. If $n$ real numbers $x_{1}, \ldots, x_{n}$ satisfy $\sum_{i=1}^{n} x_{1}=0$, then

$$
\max \left|x_{i}\right| \leqslant b_{n} \max \left|x_{1+1}-x_{1}\right|
$$

where $b_{n}=n / 4$ for even $n$ and $b_{n}=\left(n^{2}-1\right) / 4 n$ for odd $n$ and $b_{n}$ are best possible.

Remark 3.1. We can replace real $x$, by complex $z_{1}$. If $z_{1}, \ldots, z_{n}$ satisfies $\sum_{i=1}^{n} z_{i}=0$, so do $z_{i} e^{i \theta}, \ldots, z_{n} e^{i \theta}$. We now choose $\theta$ such that $\left|z_{1}\right|=z_{1} e^{i \theta}=\max \left|z_{1}\right|$ ( 0 if there is more than one $l$ possible, choose the first one) and let $x_{1} \equiv \operatorname{Re} z_{1} e^{\prime \theta}$. The sequence $x_{1}, \ldots, x_{n}$ satisfies the conditions of Theorem $\mathrm{A}$ and therefore.

$$
\max \left|x_{i}\right|=\left|z_{l}\right|=\max \left|z_{i}\right| \leqslant b_{n} \max \left|\operatorname{Re}\left(z_{1+1}-z_{1}\right) e^{i \theta}\right| \leqslant b_{n} \max \left|z_{1+1}-z_{1}\right| .
$$


We can also prove

THEOREM 3.2. For $x_{1}, \ldots, x_{n}, n$ complex numbers, $n \geqslant 2$, satisfying $\sum_{i=1}^{n} x_{i}=0$ we have $\max \left|x_{i}\right| \leqslant b_{n}^{k} \max \left|\Delta^{k} x_{i}\right|$ and $\left(\sum_{i=1}^{n}\left|x_{i}\right|^{p}\right)^{1 / p} \leqslant b_{n}^{k}\left(\sum_{i=1}^{n}\left|\Delta^{k} x_{i}\right|^{p}\right)^{1 / p}$ for $p \geqslant 1$ where $b_{n}$ is given in Theorem A, $\Delta x_{i} \equiv x_{i+1}-x_{i}, \Delta^{k} x_{i} \equiv \Delta\left(\Delta^{k-1} x_{i}\right)$ and $x_{j} \equiv x_{i}$ if $j=i(\bmod n)$.

Proof. The inequality $\max \left|x_{i}\right| \leqslant b_{n}^{k} \max \left|\Delta^{k} x_{i}\right|$ follows Theorem $\mathrm{A}$ and Remark 3.1 repreated $k$ times since $\sum_{i=1}^{n} \Delta^{\prime} x_{i}=0$. For any sequence $y_{1}, \ldots, y_{n}$ such that $\left(\sum_{i=1}^{n}\left|y_{i}\right|^{q}\right)^{1 / q}=1, p^{-1}+q^{-1}=1$, or in case $p=1, \quad \max \left|y_{i}\right|=1$ we can write $z_{i}=\sum_{j=1}^{n} x_{j+i} y_{j}$. Obviously $\sum z_{i}=\sum_{i=1}^{n} \sum_{j=1}^{n} x_{j+i} y_{j}=0$ and $\left|z_{i}\right| \leqslant\left(\sum_{j=1}^{n}\left|x_{j}\right|^{p}\right)^{1 / p}$ while $\left|\Delta^{k} z_{i}\right| \leqslant\left(\sum_{j=1}^{n}\left|\Delta^{k} x_{j}\right|^{p}\right)^{1 / p}$.

We use the first part of the proof and write

$$
\left|z_{i}\right| \leqslant b_{n}^{k} \max \left|\Delta^{k} z_{i}\right| \leqslant b_{n}^{k}\left(\sum_{j=1}^{n}\left|\Delta^{k} x_{j}\right|^{p}\right)^{1 / p},
$$

and choosing $y_{j}$ appropriately $\mid z_{i}=\left(\sum_{j=1}^{n}\left|x_{j}\right|^{p}\right)^{1 / p}$, our theorem follows.

One can prove in a similar way the following somewhat more general theorem.

THEOREM 3.3. Let $B$ be an n-dimensional Banach space of complex sequences, $x=\left(x_{1}, \ldots, x_{n}\right)$, such that $\sum_{i=1}^{n} x_{i}=0, x_{j} \equiv x_{i}, j=i(\bmod n)$ and $\left\|\left\{x_{i+1}\right\}\right\|=\left\|\left\{x_{i}\right\}\right\|$. Then $\left\|\left\{x_{i}\right\}\right\|_{B} \leqslant b_{n}^{k}\left\|\left\{\Delta^{k} x_{i}\right\}\right\|_{B}$ where $b_{n}$ is given in Theorem A.

\section{REFERENCES}

1. E. F. Beckenbach and R. Bellman, Inequalities, Springer-Verlag, Berlin and New York, 1961.

2. R. Bellman, A note on periodic functions and their derivatives, J. London Math. Soc. 18 (1943). 140-142

3. Z. Ditzian, Some remarks on inequalities of Landau and Kolmogorov, Aequationes Math. 12 (1975), |45-51.

4. K. Fan, O. Taussky and J. Todd, Discrete analogues of inequalities by Wirtinger, Monatsh. Math. Physik 59 (1955), 73-90.

5. A. Kolmogorov. On inequalities between upper hounds of the successive derivatives of an arhitrary function on an infinite interval, Amer. Math. Soc. Transl. no. 4 (1949) (Russian original 1939).

6. D. G. Northcott, Some inequalities between periodic functions and their derivatives, J. London Math Soc. 14 (1939), 198-202.

Department of Mathematics, University of Alberta, Edmonton (T6G 2G1), Canada 